\title{
Boxy/Peanut/X-Shaped Bulges: Steep Inner Rotation Curve Leads to Barlens Face-on Morphology
}

\author{
H. Salo and E. Laurikainen \\ Astronomy Research Unit, University of Oulu, FI-90014 Finland; heikki.salo@oulu.fi \\ Received 2016 May 19; revised 2016 November 8; accepted 2016 November 8; published 2017 February 1
}

\begin{abstract}
We use stellar dynamical bulge/disk/halo simulations to study whether barlenses (lens-like structures embedded in the narrow bar component) are only the face-on counterparts of Boxy/Peanut/X-shapes $(B / P / X)$ seen in edge-on bars, or if some additional physical parameter affects that morphology. A range of bulge-to-disk mass and size ratios are explored: our nominal parameters $\left(B / D=0.08, r_{\mathrm{eff}} / h_{r}=0.07\right.$, disk comprising two-thirds of total force at $2.2 h_{r}$ ) correspond to typical Milky Way mass galaxies. In all models, a bar with pronounced $\mathrm{B} / \mathrm{P} / \mathrm{X}$ forms in a few Gyr, visible in the edge-on view. However, the pure barlens morphology forms only in models with sufficiently steep inner rotation curves, $d V_{\text {cir }} / d r \gtrsim 5 V_{\max } / h_{r}$, achieved when including a small classical bulge with $B / D \gtrsim 0.02$ and $r_{\text {eff }} / h_{r} \lesssim 0.1$. For shallower slopes, the central structure still resembles a barlens, but shows a clear X signature even in low inclinations. A similar result holds for bulge-less simulations, where the central slope is modified by changing the halo concentration. The predicted sensitivity on the inner rotation curve is consistent with the slopes that are estimated from gravitational potentials calculated from the $3.6 \mu \mathrm{m}$ images, for the observed barlens and X-shaped galaxies in the Spitzer Survey of Stellar Structure in Galaxies $\left(\mathrm{S}^{4} \mathrm{G}\right)$. For inclinations $<60^{\circ}$ the galaxies with barlenses have on average twice steeper inner rotation curves than galaxies with $\mathrm{X}$ shapes: the limiting slope is $\sim 250 \mathrm{~km} \mathrm{~s}^{-1} \mathrm{kpc}^{-1}$. Among barred galaxies, those with barlenses have both the strongest bars and the largest relative excess of inner surface density, both in barlens regions $\left(\lesssim 0.5 h_{r}\right)$ and near the center $\left(\lesssim 0.1 h_{r}\right)$; this provides evidence for bar-driven secular evolution in galaxies.
\end{abstract}

Key words: galaxies: bulges - galaxies: evolution - galaxies: kinematics and dynamics - galaxies: structure

\section{Introduction}

The majority of strong bars in massive early-type disk galaxies exhibit a barlens (bl) morphology: a central lens-like structure embedded in a narrow bar component, identified as a distinct morphological feature in Laurikainen et al. (2011). Recent observational and theoretical studies support the conjecture (Laurikainen et al. 2007) that such round inner structures are in fact the face-on counterparts of the vertically extended Boxy/Peanut/X-shaped $(\mathrm{B} / \mathrm{P} / \mathrm{X})$ structures commonly seen in barred edge-on galaxies (e.g., Lütticke et al. 2000; Bureau et al. 2006). Namely, the stellar masses and the axial ratio distributions of their host galaxies are consistent with $\mathrm{B} / \mathrm{P} / \mathrm{X}$ and $\mathrm{bl}$ galaxies forming a single population (Laurikainen et al. 2014; Laurikainen \& Salo 2016a). Simulation models also show that it is possible to exhibit a $\mathrm{B} / \mathrm{P} / \mathrm{X}$ morphology in the edge-on view, while the face-on morphology is essentially circular (Athanassoula et al. 2015; Athannasoula 2016). Additional support for the $\mathrm{B} / \mathrm{P} / \mathrm{X} / \mathrm{bl}$ connection is provided by the very similar colors of the barlens and narrow bar components (Herrera-Endoqui et al. 2016). This connection of barlenses to vertically extended inner portions of bars indicates that they should not be confused with mergerrelated classical bulges, or with disky "pseudo-bulges" (i.e., inner disks).

The best examples of pronounced barlens morphology in simulations are those of Athanassoula et al. (2013), the simulations of which were analyzed in detail in the abovementioned study of Athanassoula et al. (2015). These idealized isolated galaxy $\mathrm{N}$-body $+\mathrm{SPH}$ (smoothed particle hydrodynamics) simulations followed the evolution of a stellar + gaseous disk embedded in a triaxial halo, and included recipes for converting gas into stars. The closest resemblance to observed barlens morphologies was attained with initial gas fractions of 20\%-80\% (Athanassoula et al. 2015). Recently, barlens morphology was also reported in simulations where disk galaxies were formed as a result of gas-rich mergers (Athanassoula et al. 2016). However, in most corresponding high-resolution simulations, either with or without gas (e.g., Minchev et al. 2012; Saha et al. 2012; Di Matteo et al. 2013), the formed bars have elongated shapes without round inner barlens components. In addition, a close inspection of the models of Athanassoula et al. (2015) indicates that even there, many of the simulated images (see their Figure 2) contain a slight trace of X-shaped morphology in the face-on view. Such $\mathrm{X}$-shaped signatures are rare in the observed nearly face-on barlens galaxies (Laurikainen \& Salo 2016b), suggesting that the orbital families forming the simulated central structures are not the same as those in most observed galaxies.

In this study, we perform simple stellar dynamical bulge/ disk/halo simulations to address the conditions for obtaining the observed type pure barlens morphology. We vary the parameters influencing the slope of the inner rotation curve, and show how this affects the face-on morphology of the vertically extended inner bar region. The $\mathrm{S}^{4} \mathrm{G}$ survey (Spitzer Survey of Stellar Structure in Galaxies; Sheth et al. 2010) is used as a guide for choosing the simulations parameters, and also as a comparison sample to check the predictions of the simulations.

\section{Simulations}

We perform stellar dynamical $N$-body simulations with GADGET-2 (Springel \& Hernquist 2005). The self-consistent initial galaxy models are constructed with the GalactICS software (Kuijken \& Dubinski 1995), and they consist of an 

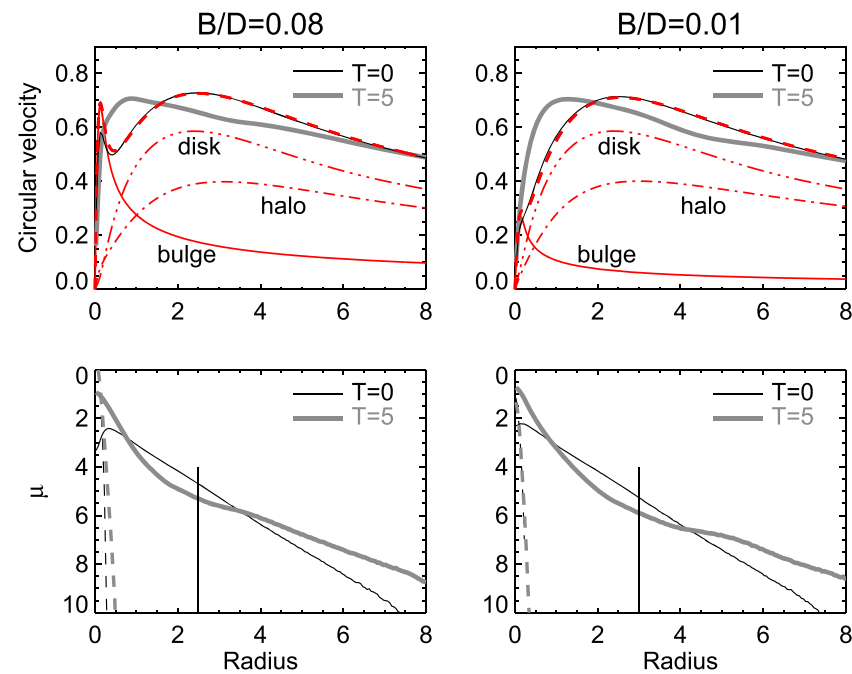

Figure 1. Simulation models. The upper row compares the rotation curves in two simulations with bulge-to-disk mass ratios $B / D=0.08$ (left) and $B / D=0.01$ (right). The red curves indicate the initial total rotation curve model from GalactICS, together with separately shown bulge, disk, and halo contributions. The black curves are the actual total circular velocity curve corresponding to the softened forces calculated by GADGET-2; the thin curve refers to the initial state, and the thick curve is after $5 \mathrm{Gyr}$. The radius is indicated in units of initial disk scale length; one velocity unit equals $\sim 300 \mathrm{~km} \mathrm{~s}^{-1}$ when using the scaling to physical units discussed in the text. The lower frames show the azimuthally averaged surface densities (in magnitude units, with arbitrary zero-point) of the disk (solid lines) and bulge (dashed lines). Again, the thin lines refer to the initial model, whereas the thick lines are after 5 Gyr.

exponential disk and a lowered Evans model (truncated logpotential with a core) for the spherical halo. Compared to the $N$-body-SPH simulations in Athanassoula et al. (2013), our simulations contain no gas or star formation; we also include a small classical bulge (King model) in the initial conditions, whereas in their study, central components could form during the simulation via gas inflow and star formation.

Our nominal model parameters are based on the analysis of the $3.6 \mu \mathrm{m} \mathrm{S}^{4} \mathrm{G}$ images. To mimic a typical Milky Way (MW) galaxy (with stellar mass $M^{*} \sim 5 \cdot 10^{10} M_{\odot}$ ), we use a bulgeto-disk mass ratio $B / D=0.08$ and a bulge effective radius $r_{\text {eff }} / h_{r}=0.07$, where $h_{r}$ denotes the scale length of the disk; these were typical bulge values obtained for Hubble types $T=3$ in the $S^{4} G$ pipeline bulge/disk/bar decompositions (Salo et al. 2015). More detailed decomposition, using a separate component for a bar and a barlens (Laurikainen et al. 2014), indicated similar small $B / D$ values, even for galaxies with $-2 \leqslant T \leqslant 0$. Our halo model is chosen so that at $2.2 h_{r}$, the disk accounts for $65 \%$ of the total radial force. This corresponds to the typical value estimated for an MW mass galaxy in Díaz-García et al. (2016b), obtained by combining the gravitational field calculated from the $3.6 \mu \mathrm{m}$ images using the NIRQB code (Salo et al. 1999; Laurikainen \& Salo 2002), with the rotation amplitudes obtained from the HI-kinematics (Courtois et al. 2009). The initial Toomre parameter $Q_{T} \approx 1.35$ at $2.2 h_{r}$, and the vertical thickness of the disk have a constant scale height $z_{o}=0.2 h_{r}$, which corresponds to the ratio of vertical to radial velocity dispersion $\sigma_{z} / \sigma_{r} \approx 0.6$ at $2.2 h_{r}$.

The simulation units are fixed by setting the gravitational constant, the mass, and the initial scale length of the disk to unity. To convert to physical units, we identify $m_{D}=$
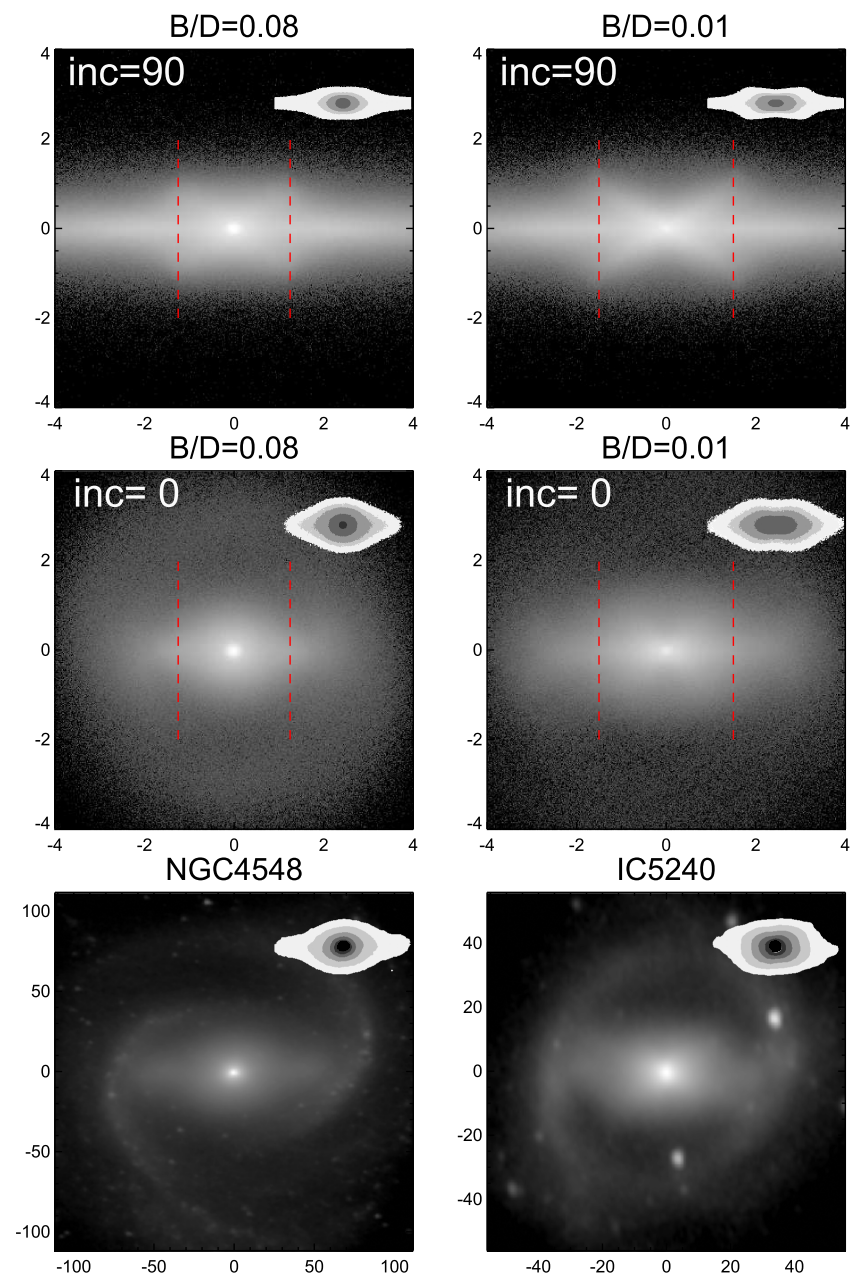

Figure 2. Two simulation models with a small classical bulge: $B / D=0.08$ (left) and $B / D=0.01$ (right). Both lead to a $\mathrm{B} / \mathrm{P} / \mathrm{X}$ shape structure when seen edge-on ( $\mathrm{i}=90^{\circ}$, upper row). In the face-on view ( $\mathrm{i}=0^{\circ}$, middle) the $B / D=0.08$ simulation has a morphology similar to the observed barlens galaxies, whereas the model with $B / D=0.01$ shows a trace of the X-shaped signature. The difference is particularly clear in the small insert figures, showing the isophotal contours of the central regions. The lowermost row shows two deprojected images from the $\mathrm{S}^{4} \mathrm{G}$-survey, NGC $4548\left(\mathrm{i}=39^{\circ}\right.$, with a barlens), and IC 5240 ( $\mathrm{i}=44^{\circ}$, with an $\mathrm{X}$ shape). In the upper two rows, the axis units are in simulation units (initial scale lengths), and those in the lowermost row are in arcseconds.

$5 \cdot 10^{10} M_{\odot}$ and $h_{r}=2.5 \mathrm{kpc}$, which correspond to the maximum disk contribution to circular velocity $\sim 170 \mathrm{~km} \mathrm{~s}^{-1}$ (total circular velocity $\sim 215 \mathrm{~km} \mathrm{~s}^{-1}$; see Figure 1). Our standard value for gravity softening is $\epsilon=0.01 h_{r}$, and the time step is $\sim 10^{-2}$ time units; in physical units one time unit corresponds to $\sim 10 \mathrm{Myr}$. The disk is produced with $10^{6}$ particles, each with a mass of $5 \cdot 10^{4} M_{\odot}$. The halo and bulge particles have the same mass as the disk particles.

The rotation curve and the disk and bulge surface density profiles of our nominal simulation model with $B / D=0.08$ are displayed in Figure 1, both for the initial state and for $T=5 \mathrm{Gyr}$, after a bar has formed. An otherwise similar model with $B / D=0.01$ is also shown. As expected (see, e.g., Debattista et al. 2006), the formation of the bar modifies the disk density profile, which becomes more centrally concentrated, whereas the scale length of the outer disk increases. This 
a)

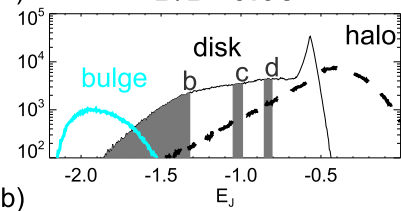

b)

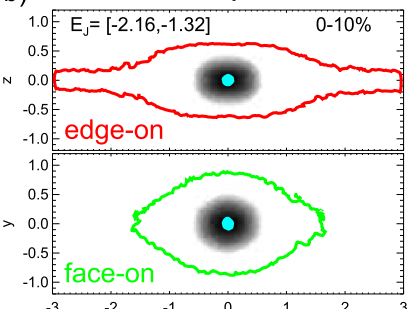

c) $\begin{array}{cccccc}-3 & -2 & -1 & 0 & 1 & 2 \\ 1.0 & E_{1}=[-1.06-0.98] & & & 25-30 \%\end{array}$

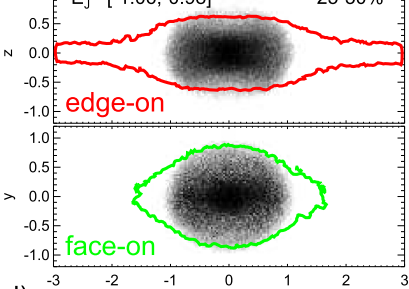

d) ${ }_{10}^{-3}$\begin{tabular}{ccccc}
-2 & -1 & 0 & 1 & 2 \\
\hline & $E_{1}=[-0.86,-0.80]$ & & & $40-45 \%$
\end{tabular}

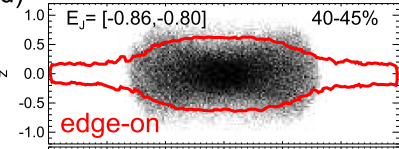

-1.0 edge-on

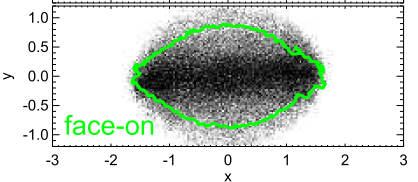

Figure 3. Orbital structure in $B / D=0.08$ (left column) and $B / D=0.01$ (right column) simulations. The uppermost row (a) shows the distribution of Jacobi energy $E_{J}$ : the bulge, disk, and halo components are shown separately. The frames (b)-(d) show the time-averaged density of orbits (between 10 and $11 \mathrm{Gyr}$ ), for the disk particles falling in the three $E_{j}$ ranges, indicated by the shaded areas in the histograms of (a): the upper frame gives an edge-on view and the lower frame gives a face-on view. Thick contours outline the edge-on (red) and face-on (green) bar morphology. The blue filled circle in (b) indicates where the projected surface density of the classical bulge exceeds that of the disk.

also modifies the rotation curve, although the central slopes are little affected. In all the following, the discussed rotation curves correspond to those after the bar has already formed.

Figure 2 compares the morphology in simulations with two different bulge-to-disk mass ratios, $B / D=0.08$ (left) and $B / D=0.01$ (right). Both simulations form a bar during $T=1-2$ Gyr, and while the bar starts to form, the vertical extent of the orbits in the inner part of the bar increases. At $T=5 \mathrm{Gyr}$ (shown in the figure), in both cases, this has resulted in a pronounced $\mathrm{B} / \mathrm{P} / \mathrm{X}$ edge-on morphology. The vertical evolution lacks the rapid buckling phase often reported in the literature (Raha et al. 1991), in which sense it is more reminiscent of the resonance heating models (Quillen et al. 2014), where the $X$ is associated with disk stars heated by the 2:1 vertical resonance (Combes et al. 1990; Pfenniger \& Friedli 1991; Patsis et al. 2002). Most importantly for the current study, the face-on morphology for $B / D=0.08$ is similar to that in massive barred galaxies, with a dominant, round barlens structure and a weak, thin bar component (compare to NGC 4548 in the lowermost row; see Laurikainen
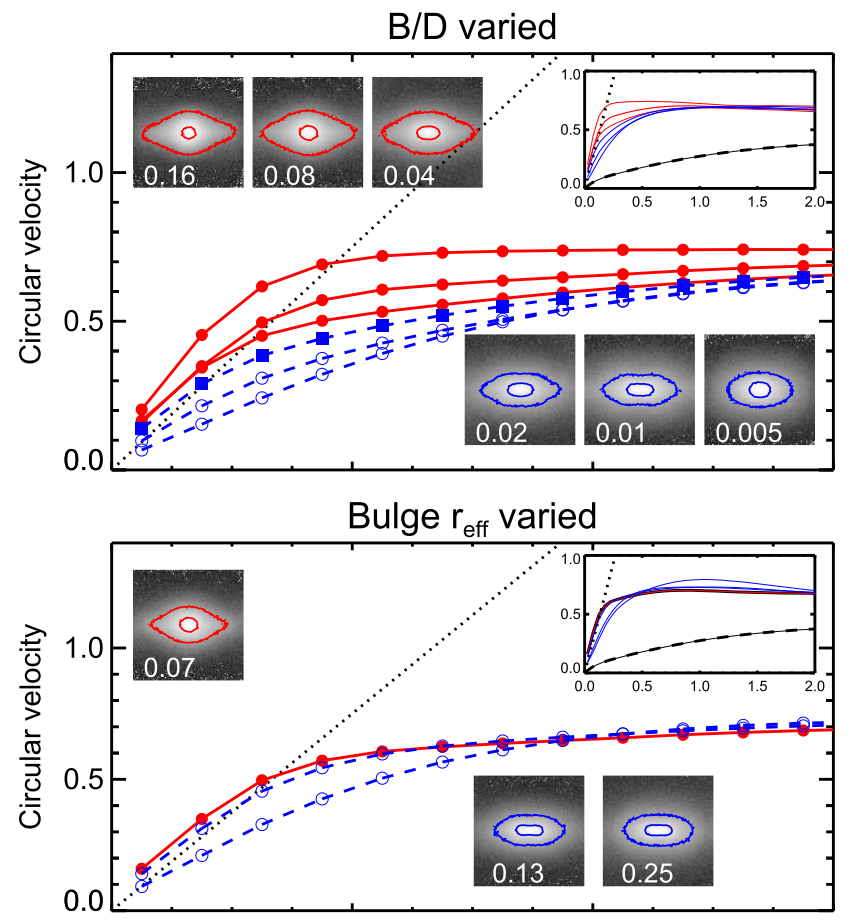

Halo concentration varied

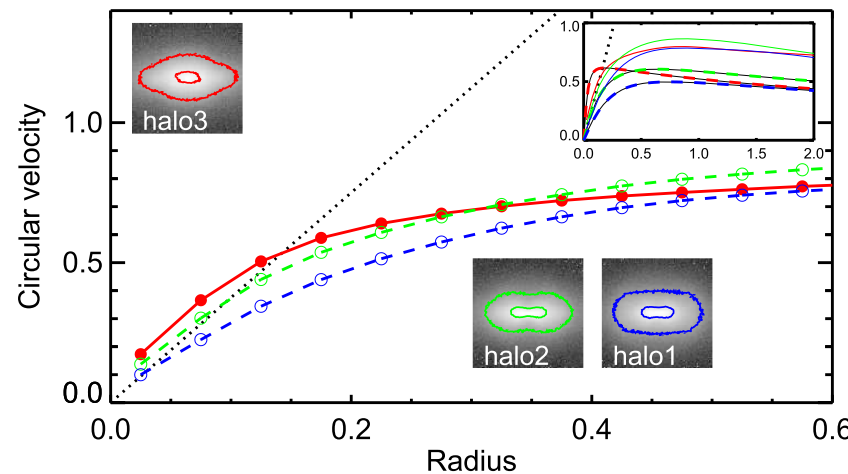

Figure 4. Central slope of the circular velocity curve in simulations with different $B / D=0.005-0.16$ (uppermost frame; $r_{\text {eff }} / h_{r}$ fixed to 0.07 ) and different bulge $r_{\text {eff }} / h_{r}=0.07-0.25$ (middle frame; $B / D$ fixed to 0.08 ), and in bulge-less simulations with different degrees of halo concentration (lowermost frame; halo concentration increases from halo model "halo1" to "halo3"); red curves indicate simulations leading to a pure barlens morphology (round inner morphology with no trace $\mathrm{X}$ in the face-on view). The dashed line indicates the approximate slope separating the morphologies $\left(d V_{\text {cir }} / d r=5 V_{\max } / h_{r}\right)$. The insert figures display the rotation curve on a larger scale, with the halo contribution marked with a dashed line; the insert snapshots show the barlens morphology (here using only the disk particles). The rotation curve corresponds to $T=5 \mathrm{Gyr}$; however, a practically similar threshold would apply when using the rotation curves from the time before the bar has formed.

\& Salo (2016b) for classification of observed barlens categories): this structure survived to the end of the simulation (12 Gyr). In the face-on view, the size of the barlens, and that of the X feature in edge-on view, are about one-half of the bar size, similar to what was found in Athanassoula et al. (2015). In the run with $B / D=0.01$, the face-on morphology also resembles a barlens, but with a clear waist-like narrowing at the bar minor axis. Most of the simulations in Athanassoula et al. (2015), as well as that in Figure 1 in Laurikainen et al. (2014), resemble more the simulation with $B / D=0.01$ than that with $B / D=0.08$. Although such traces of $\mathrm{X}$-like 

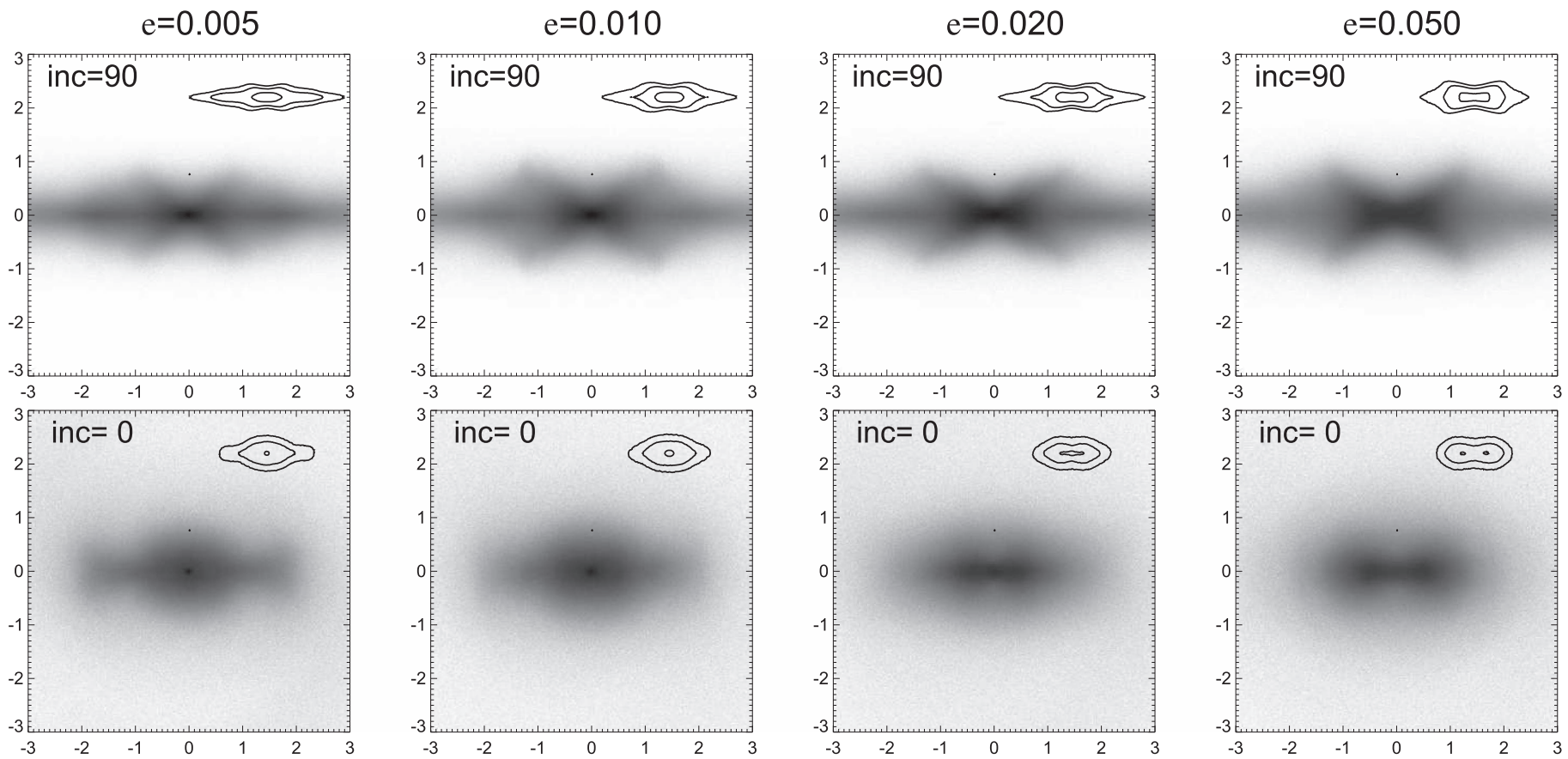

Figure 5. Effect of softening in the simulated inner morphology (total extent of the frames is 6 simulation units). From left to right the softening increases from $\epsilon / h_{r}=0.005$ to 0.05 . Even a twofold softening compared to the nominal value 0.01 destroys the barlens morphology, whereas with a smaller softening, an even better resemblance to the observed pure barlens morphology is attained.

morphology are not often seen in observed nearly face-on galaxies, one example is IC 5240, with an inclination of $\mathrm{i}=44^{\circ}$.

The difference in the orbital structure of the two preceding simulations is illustrated in Figure 3, showing the timeaveraged density distributions of selected disk particle orbits, in a frame co-rotating with the bar. The plots were constructed by sampling the positions of all particles at 500 equally separated instants during the time interval $T=10-11 \mathrm{Gyr}$, when the bar pattern speed $\Omega_{\text {bar }}$ had attained a constant value (within a few percents). The bar pattern speed was measured by calculating the moment of inertia tensor for all disk particles within 3 distance units from the center, and following the direction of its longest principal axis; the mean rate of change over the time interval was expressed as $\Omega_{\mathrm{bar}}$. The positions of particles were converted to the rotating system and tabulated in twodimensional bins in $x y$ and $x z$ planes, where $x$ denotes the coordinate along the bar major axis. The tabulations from all 500 sampling times were added together, yielding the face-on and edge-on projections of the time-averaged density of the selected orbits. We also calculated the Jacobi energies of the particles, $E_{j}=\frac{1}{2} v^{2}+\Phi-\frac{1}{2} \Omega_{\mathrm{bar}}^{2}\left(x^{2}+y^{2}\right)$, where $\Phi$ is the gravitational potential and $v$ is the velocity in the rotating frame; over the sampling interval, the $E_{j}$ of particles stayed nearly constant.

In Figure 3, the orbits of disk particles in three different ranges of Jacobi energy are shown separately in frames (b), (c), and $(\mathrm{d})$; the ranges are indicated at the $E_{j}$ histogram in frame (a). In the $B / D=0.08$ run (left frames), the orbits of the disk particles with $E_{J}$ in the lowest $10 \%$ range (frame b, left) fill a nearly circular region in the face-on projection. For the same $E_{J}$ range, in the $B / D=0.01$ run (frame $\mathrm{b}$, right), the density distribution is clearly elongated along the bar major axis. A similar difference concerns the orbits in the energy range
$25 \%-30 \%$ (frame c), which more or less outlines the circular barlens in the $B / D=0.08$ case, and in the energy range $40 \%-$ $45 \%$ (frame $\mathrm{d}$ ), comprising the orbits with the largest vertical extent. For the $B / D=0.01$ model, the orbits in frames (c) and (d) show a clear box-orbit character, with pronounced narrowing in the bar minor axis direction. Clearly, a significant central mass concentration can affect the orbital structure in a significant portion of the bar, favoring loop-like orbits responsible for the round face-on morphology.

The fundamental difference in the above $B / D=0.08$ and $B / D=0.01$ simulations is the much higher central density in the former model, leading to a significantly steeper inner rotation curve. The effect of the inner rotation curve is further illustrated in Figure 4 (uppermost frame), displaying a range of $B / D$ ratios from 0.005 to 0.16 , whereas the bulge-effective radius is kept fixed to $r_{\text {eff }}=0.07 h_{r}$. In these experiments, a hint of X-shaped face-on morphology (manifested in isophotes as a waist in the minor axis; see the insert figures) appears when the central slope $d V_{\text {cir }} / d r \lesssim 5 V_{\max } / h_{r}$ (occurs for $B / D \lesssim 0.02$ ). The same threshold applies to models in which the inner slope is reduced by increasing the bulge effective radius: for example, doubling the effective radius to $r_{\mathrm{eff}}=0.13 h_{r}$ for $B / D=0.08$, yields a clear X signature (middle frame). Similar dependence on rotation curve slope is also seen in models that have no classical bulge component, when the degree of halo central concentration is varied (lowermost frame). Note that the rotation curve is displayed at the time when the bar has formed ( $T \sim 5 \mathrm{Gyr}$ ); the initial $h_{r}$ is used here mainly to get a rough normalization of the slopes. During the formation of the bar, the outer disk scale length typically increases by a factor of $50 \%-100 \%$ (see Figure 1).

Because the simulated morphology is sensitive to the steepness of the central velocity slope, even the use of too large a gravity softening may prevent the pure barlens 
morphology. This is illustrated in Figure 5, for the concentrated halo model ("halo3") of Figure 4, which with our nominal softening $\left(\epsilon / h_{r}=0.01\right)$ leads to barlens morphology. Doubling the softening value $\left(\epsilon / h_{r}=0.02\right)$ leads to clearly elongated central structure. In contrast, reducing $\epsilon / h_{r}$ to 0.005 yields a nearly similar pure barlens morphology as the nominal value. For our model with $B / D=0.08$, a similar change of morphology takes place between $\epsilon / h_{r}=0.02$ and 0.05 . Interestingly, the edge-on morphology is much less affected by increased softening: since most previous simulations of $\mathrm{B} / \mathrm{P} / \mathrm{X}$ structure have concentrated on the bar vertical structure, the dependence of face-on morphology on softening may have escaped attention.

\section{Comparison to Observations}

The preceding simulations suggest that there should be a more or less clear-cut difference (without/with trace of $\mathrm{X}$ ) in the inner morphology for barlens galaxies with steep/shallow inner rotation curve slopes, respectively. Can this be verified with observations? Unfortunately, the number of barlens galaxies with reliably measured rotation curves is very small: for example, a recent study of inner rotation curves based on high-resolution $\mathrm{H}_{\alpha}$ kinematics of $29 \mathrm{~S}^{4} \mathrm{G}$ galaxies (Erroz-Ferrer et al. 2016) contains only two galaxies in common with the compilation of barlens and $\mathrm{X}$-shaped galaxies in Laurikainen et al. (2014). However, Erroz-Ferrer et al. (2016) also conclude that the inner rotation curves are dominated by baryonic matter (see also Lelli et al. (2013)), based on a comparison of inner slopes of $\mathrm{H}_{\alpha}$ rotation curves with those derived from the $\mathrm{S}^{4} \mathrm{G}$ mass maps (nonstellar contaminants removed based on both 3.6 and $4.5 \mu \mathrm{m}$ images; Querejeta et al. 2015) using the abovementioned NIRQB code. We may therefore approximate the inner rotation curve slopes with those derived from the mid-IR images: this is done in Figure 6 for all barlens and X-shaped galaxies in $S^{4} G$. Because the rotation curves calculated from the raw $3.6 \mu \mathrm{m} \mathrm{S}{ }^{4} \mathrm{G}$ images and the mass maps differ very little (Díaz-García et al. 2016b), the slopes in the figure are based on raw $3.6 \mu \mathrm{m}$ images. The median ratio between the observed slopes in Erroz-Ferrer et al. (2016) and our estimated slopes is 0.96 (using the 24 galaxies with observed slopes given in Table 3 of Erroz-Ferrer et al. 2016).

Figure 6 (upper frame) displays the inner rotation curve slopes (in units of $\mathrm{km} \mathrm{s}^{-1} \mathrm{kpc}^{-1}$ ) versus galaxy stellar mass. The slopes of barlens galaxies are on average a factor of two steeper than slopes with X-shaped structures. In addition, the insert figure indicates a possible tendency for a barlens to be rounder when the central slope is steeper, which is in accordance with the simulated behavior. Although the fit is not statistically significant, this dependence on slope is interesting because in Laurikainen et al. (2014) and Athanassoula et al. (2015), no trend was found between the axial ratio of the barlens and the bar strength. Because the visibility of possible weak X signatures depends strongly on the observing direction (Laurikainen \& Salo 2016b), the lower frame makes a comparison as a function of galaxy inclination. Furthermore, to eliminate the influence of galaxy mass, the slopes are normalized by $V_{\max } / h_{r}$. Excluding the highly inclined galaxies with $i \gtrsim 60^{\circ}$ (which are all $\mathrm{X}$-shaped), the separation between barlens and X-shaped parent galaxies is strikingly clear, and in good qualitative agreement with the simulation predictions. In the figure, the division between pure barlens and the lowinclination $\mathrm{X}$ shape is drawn at $250 \mathrm{~km} \mathrm{~s}^{-1} \mathrm{kpc}^{-1}$ and at 4
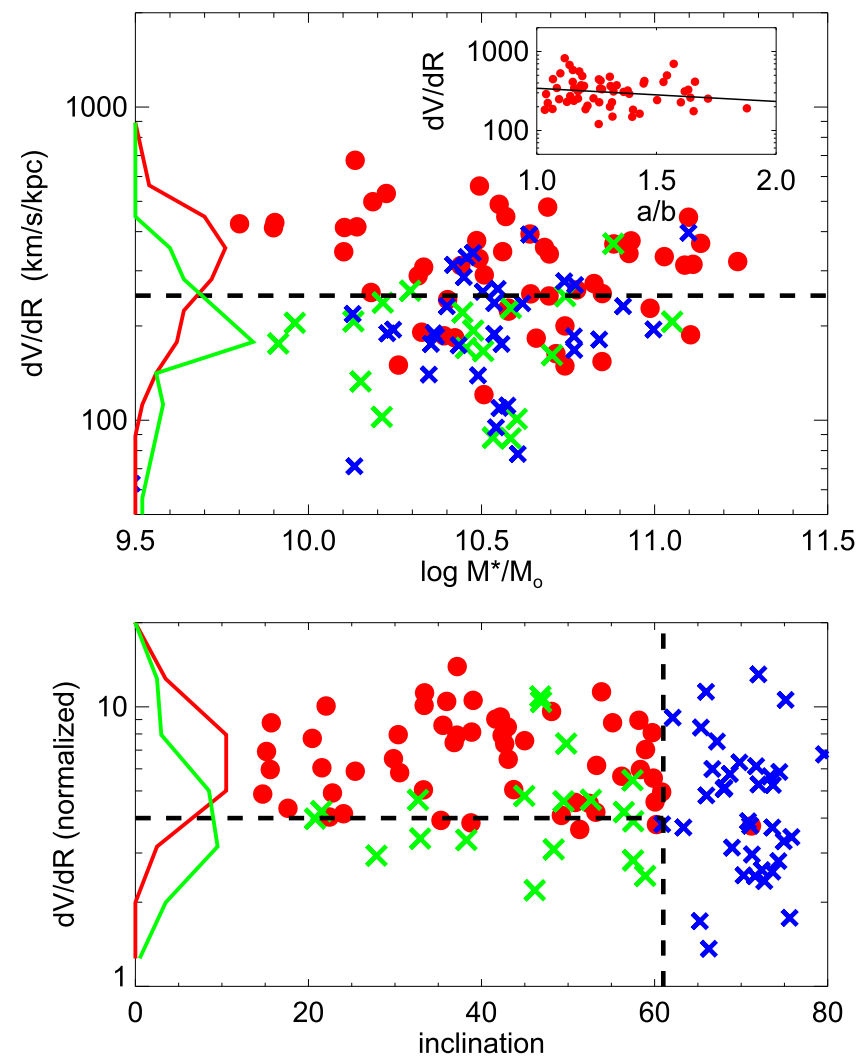

Figure 6. Slopes of the inner rotation curves for $S^{4} \mathrm{G}$ galaxies, calculated from the $3.6 \mu \mathrm{m}$ images. The red circles indicate galaxies with a barlens identification (Laurikainen et al. 2011; Buta et al. 2015), whereas green $\left(i<60^{\circ}\right)$ and blue crosses $\left(i>60^{\circ}\right)$ represent galaxies with an $\mathrm{X}$ signature. In the upper frame the inner slope is shown vs. galaxy stellar mass ( $M^{*}$ from Munoz-Mateos et al. 2015), whereas in the lower frame, the slope is normalized by $V_{\max } / h_{r}$ and displayed vs. galaxy inclination. Here $V_{\max }$ is the calculated maximum velocity due to visible mass and $h_{r}$ is the outer disk scale length from Salo et al. (2015). All parameter values used in Figure 6 are also listed in Table 1. Histograms in the left show the distributions of barlens and Xshaped parent galaxies; they cover all inclinations, so that both prominent edgeon $\mathrm{X}$ shapes and weaker $\mathrm{X}$-like signatures at small and intermediate inclinations are included. The insert in the upper frame shows the central velocity slope vs. barlens axial ratio (deprojected to the disk plane).

$V_{\max } / h_{r}$. The latter is close to simulation predictions; note, however, that here $V_{\max }$ refers to stellar-only contributions, and $h_{r}$ is the observed disk scale length-after the bar has formed.

Three galaxies with X-shaped bars-NGC 4123, NGC 4725, and NGC 7140-appear in the barlens region in Figure 6, lower frame, where the normalized $d V_{\text {cir }} / d r$ is used (they have $i<60^{\circ}$ and $d V_{\text {cir }} / d r>8 V_{\max } / h_{r}$; see Table 1$)$. For NGC 4725 this is the case also when expressing the central slope in $\mathrm{km} \mathrm{s}^{-1} \mathrm{kpc}^{-1}$ (upper frame). In NGC 4725, most likely a large fraction of the central mass concentration inside the $\mathrm{X}$ feature comes from a nuclear bar. In NGC 7140, it is largely due to a nuclear ringlens, and in NGC 4123, it is due to a point-like nucleus. All these nuclear features are expected to have formed secularly from the disk material. There is no clear indication of why these three galaxies deviate from the common trend; this might be related to factors not addressed by the simulations, such as the gas content or the halo-to-disk mass ratio.

The morphology of the preceding simulation models is further studied in Laurikainen \& Salo (2016b), where we demonstrate that the $B / D=0.08$ model, when viewed from different inclinations and orientations with respect to the bar 
Table 1

Data for Galaxies with Barlens and X Features Used in Figure 6

\begin{tabular}{|c|c|c|c|c|c|c|c|}
\hline Galaxy & Hubble Type & $\begin{array}{c}d V_{\text {cir }} / d r \\
\left(\mathrm{~km} \mathrm{~s}^{-1} / \mathrm{kpc}^{-1}\right)\end{array}$ & $\begin{array}{c}d V_{\text {cir }} / d r \\
\text { normalized }\end{array}$ & $\begin{array}{c}V_{\max } \\
\left(\mathrm{km} \mathrm{s}^{-1}\right)\end{array}$ & $\begin{array}{c}h_{r} \\
(\mathrm{kpc})\end{array}$ & $\begin{array}{c}\text { INC } \\
\text { (degrees) }\end{array}$ & $\log _{10}\left(M^{*} / M_{\odot}\right)$ \\
\hline \multicolumn{8}{|c|}{ Galaxies with barlenses } \\
\hline IC 2051 & $\mathrm{SB}(\underline{\mathrm{r} s}, \mathrm{bl}) \mathrm{b}$ & 223 & 4.20 & 155 & 2.92 & 53.2 & 10.58 \\
\hline NGC 0613 & $\mathrm{SB}(\underline{\mathrm{r} s} \mathrm{~s}, \mathrm{bl}, \mathrm{nr}) \mathrm{b}$ & 312 & 8.12 & 211 & 5.50 & 38.9 & 11.09 \\
\hline NGC 0936 & (L) $\mathrm{SB}_{\mathrm{a}}(\underline{\mathrm{r}} \mathrm{s}, \mathrm{bl}) 0^{+}$ & 340 & 7.90 & 188 & 4.37 & 42.4 & 10.93 \\
\hline NGC 1015 & $\left(\mathrm{R}^{\prime}\right) \mathrm{SB}(\mathrm{r}, \mathrm{bl}) 0 / \mathrm{a}$ & 186 & 5.81 & 123 & 3.87 & 30.5 & 10.39 \\
\hline NGC 1022 & (RL)SAB(rss,bl,ns) $\underline{0} / \mathrm{a}$ & 498 & 6.05 & 153 & 1.86 & 21.6 & 10.19 \\
\hline NGC 1079 & $(\mathrm{R} \underline{L}) \mathrm{S}\left(\underline{\mathrm{A}} \mathrm{B}_{\mathrm{a}}(\underline{\mathrm{r}} \mathrm{s}, \mathrm{bl}) 0\right.$ & 227 & 4.52 & 145 & 2.88 & 52.4 & 10.58 \\
\hline NGC 1097 & $\left(\mathrm{R}^{\prime}\right) \mathrm{SB}(\mathrm{rs}, \mathrm{bl}, \mathrm{nr}) \mathrm{ab}$ pec & 321 & 9.63 & 265 & 7.96 & 48.1 & 11.24 \\
\hline NGC 1300 & $\left(\mathrm{R}^{\prime}\right) \mathrm{SB}(\mathrm{s}, \mathrm{bl}, \mathrm{nrl}) \mathrm{b}$ & 227 & 10.12 & 122 & 5.45 & 33.4 & 10.58 \\
\hline NGC 1302 & (RLRL)SAB(rl, bl) $0^{+}$ & 345 & 8.77 & 153 & 3.89 & 15.7 & 10.56 \\
\hline NGC 1326 & $\left(\mathrm{R}_{1}\right) \mathrm{SAB}_{\mathrm{a}}(\mathrm{r}, \mathrm{bl}, \mathrm{nr}) 0^{+}$ & 488 & 7.92 & 190 & 3.09 & 37.2 & 10.55 \\
\hline NGC 1350 & $(\mathrm{R}) \mathrm{SAB}_{\mathrm{a}}(\mathrm{r}, \mathrm{bl}) 0 / \mathrm{a}$ & 273 & 8.95 & 158 & 5.18 & 58.2 & 10.82 \\
\hline NGC 1398 & $\left(\mathrm{R}^{\prime} \mathrm{R}\right) \mathrm{SB}(\underline{\mathrm{r}} \mathrm{s}, \mathrm{bl}) \mathrm{a}$ & 367 & 9.19 & 222 & 5.57 & 42.2 & 11.13 \\
\hline NGC 1452 & $(\mathrm{RL}) \mathrm{SB}(\underline{\mathrm{r} s}, \mathrm{bl}) 0 / \mathrm{a}$ & 242 & 6.17 & 133 & 3.38 & 53.3 & 10.40 \\
\hline NGC 1512 & $(\mathrm{RL}) \mathrm{SB}(\mathrm{r}, \mathrm{bl}, \mathrm{nr}) \mathrm{a}$ & 308 & 7.37 & 116 & 2.79 & 42.7 & 10.33 \\
\hline NGC 1533 & $(\mathrm{RL}) \mathrm{SB}(\mathrm{bl}) 0^{\circ}$ & 447 & 5.97 & 175 & 2.34 & 15.6 & 10.57 \\
\hline NGC 1640 & $\left(\mathrm{R}^{\prime}\right) \mathrm{SB}_{\mathrm{a}}(\mathrm{r}, \mathrm{bl}) \mathrm{ab}$ & 255 & 4.14 & 114 & 1.85 & 24.1 & 10.18 \\
\hline NGC 2543 & $\mathrm{SAB}(\mathrm{s}, \mathrm{bl}) \mathrm{b}$ & 183 & 5.56 & 119 & 3.61 & 59.9 & 10.42 \\
\hline NGC 2787 & $(\mathrm{~L}) \mathrm{SB}_{\mathrm{a}}(\mathrm{r}, \mathrm{bl}) 0^{\circ}$ & 529 & 5.64 & 148 & 1.59 & 56.2 & 10.23 \\
\hline NGC 2859 & $(\mathrm{R}) \mathrm{SAB}_{\mathrm{a}}(\mathrm{rl}, \mathrm{bl}, \mathrm{nl}, \mathrm{nb}) 0^{+}$ & 365 & 13.93 & 215 & 8.20 & 37.2 & 10.88 \\
\hline NGC 2968 & $(\mathrm{~L}) \mathrm{SB}(\mathrm{s}, \mathrm{bl}) 0^{+}$ & 412 & 6.49 & 126 & 1.99 & 43.1 & 10.10 \\
\hline NGC 3351 & $\left(\mathrm{R}^{\prime}\right) \mathrm{SB}(\mathrm{r}, \mathrm{bl}, \mathrm{nr}) \mathrm{a}$ & 374 & 7.57 & 140 & 2.85 & 45.0 & 10.49 \\
\hline NGC 3384 & (L)SAB $\underline{\mathrm{B}}(\mathrm{bl}) 0^{-}$ & 328 & 4.96 & 156 & 2.37 & 60.8 & 10.49 \\
\hline NGC 3489 & $(\mathrm{R}) \mathrm{SA} \underline{\mathrm{B}}(\mathrm{r}, \mathrm{bl}) 0^{\circ}$ : & 676 & 4.57 & 146 & 0.99 & 60.1 & 10.14 \\
\hline NGC 3941 & $(\mathrm{R}) \mathrm{SB}_{\mathrm{a}}(\mathrm{bl}) 0^{\circ}$ & 559 & 4.55 & 179 & 1.46 & 50.8 & 10.49 \\
\hline NGC 3953 & $\mathrm{SB}(\mathrm{r}, \mathrm{bl}) \mathrm{b}$ & 227 & 5.97 & 162 & 4.25 & 58.4 & 10.99 \\
\hline NGC 3992 & $\mathrm{SB}(\mathrm{rs}, \mathrm{bl}, \mathrm{nb}) \mathrm{ab}$ & 187 & 8.77 & 173 & 8.10 & 55.1 & 11.10 \\
\hline NGC 4245 & $(\mathrm{RL}) \mathrm{SB}(\mathrm{r}, \mathrm{bl}, \mathrm{nr} \mathrm{l}) 0^{+}$ & 423 & 5.05 & 95 & 1.13 & 33.3 & 9.80 \\
\hline NGC 4314 & $\left(\mathrm{R}_{1}{ }^{\prime}\right) \mathrm{SB}(\mathrm{rl}, \mathrm{bl}, \mathrm{nr}) \mathrm{a}$ & 414 & 7.71 & 118 & 2.20 & 20.4 & 10.14 \\
\hline NGC 4371 & (L)SBa $(\mathrm{r}, \mathrm{bl}, \mathrm{nr}) 0^{+}$ & 291 & 7.03 & 145 & 3.52 & 59.0 & 10.51 \\
\hline NGC 4394 & $(\underline{\mathrm{R} L}) \mathrm{SB}(\mathrm{rs}, \mathrm{bl}, \mathrm{nl}) 0 / \mathrm{a}$ & 311 & 7.95 & 116 & 2.97 & 30.4 & 10.44 \\
\hline NGC 4448 & $(\mathrm{R}) \mathrm{SB}(\mathrm{r}, \mathrm{bl}) 0 / \underline{\mathrm{a}}$ & 153 & 3.75 & 162 & 3.95 & 71.2 & 10.85 \\
\hline NGC 4454 & (RL)SAB(r,bl)0/a & 182 & 4.33 & 143 & 3.39 & 17.6 & 10.66 \\
\hline NGC 4548 & $\mathrm{SB}(\mathrm{rs}, \mathrm{bl}) \underline{\mathrm{a}} \mathrm{b}$ & 339 & 10.55 & 141 & 4.39 & 39.0 & 10.70 \\
\hline NGC 4579 & $\left(\underline{\mathrm{R}} \mathrm{LR}^{\prime}\right) \mathrm{SB}(\mathrm{rs}, \mathrm{bl}) \mathrm{a}$ & 444 & 9.04 & 211 & 4.30 & 41.6 & 11.10 \\
\hline NGC 4593 & $\left(\mathrm{R}^{\prime}\right) \mathrm{SB}(\mathrm{rs}, \mathrm{bl}, \mathrm{AGN}) \mathrm{a}$ & 373 & 11.22 & 212 & 6.38 & 33.4 & 10.93 \\
\hline NGC 4596 & (L)SB(rs,bl) $\underline{0} / \mathrm{a}$ & 356 & 8.59 & 159 & 3.84 & 35.5 & 10.68 \\
\hline NGC 4639 & $\left(\mathrm{R}^{\prime}\right) \mathrm{SA} \underline{\mathrm{B}}(\mathrm{rs}, \mathrm{bl}) \mathrm{ab}$ & 289 & 4.09 & 134 & 1.90 & 49.2 & 10.32 \\
\hline NGC 4643 & (L) $\mathrm{SB}(\underline{\mathrm{rs}} \mathrm{s}, \mathrm{bl}, \mathrm{nl}) 0^{+}$ & 332 & 7.45 & 228 & 5.11 & 36.8 & 11.03 \\
\hline NGC 4754 & (L) $\mathrm{SB}_{\mathrm{a}}(\mathrm{bl}) 0^{\circ}$ & 393 & 8.08 & 167 & 3.43 & 59.7 & 10.64 \\
\hline NGC 4795 & $\left(\mathrm{R}^{\prime}\right) \mathrm{SA} \underline{B}_{\mathrm{a}}(1, \mathrm{bl}) \mathrm{a}$ pec & 200 & 5.05 & 157 & 3.98 & 43.7 & 10.74 \\
\hline NGC 4984 & $\left(\mathrm{R}^{\prime} \mathrm{R}\right) \mathrm{SAB}_{\mathrm{a}}(1, \mathrm{bl}, \mathrm{nl}) 0 / \mathrm{a}$ & 479 & 11.31 & 217 & 5.14 & 53.9 & 10.69 \\
\hline NGC 5101 & $\left(\mathrm{R}_{1} \mathrm{R}_{2}^{\prime}\right) \mathrm{SB}(\underline{\mathrm{r} s}, \mathrm{bl}) 0 / \mathrm{a}$ & 314 & 10.07 & 218 & 6.99 & 22.0 & 11.11 \\
\hline NGC 5134 & (R)SAB(rs,bl)a & 411 & 4.87 & 103 & 1.23 & 14.7 & 9.90 \\
\hline NGC 5339 & SAB $\underline{B}(r s, b l) a b$ & 120 & 3.84 & 123 & 3.94 & 38.8 & 10.51 \\
\hline NGC 5347 & $\mathrm{SB}(\mathrm{rs}, \mathrm{bl}) \mathrm{a}$ & 427 & 4.92 & 174 & 2.01 & 22.8 & 9.90 \\
\hline NGC 5375 & $\left(\mathrm{R}^{\prime}\right) \mathrm{SB}_{\mathrm{a}}(\mathrm{rs}, \mathrm{bl}) \underline{\mathrm{a}} \mathrm{b}$ & 163 & 6.52 & 138 & 5.54 & 29.8 & 10.72 \\
\hline NGC 5701 & $\left(\mathrm{R}_{1}{ }^{\prime}\right) \mathrm{SA} \underline{\mathrm{B}}(\mathrm{rl}, \mathrm{bl}) 0 / \mathrm{a}$ & 249 & 6.92 & 162 & 4.51 & 15.2 & 10.69 \\
\hline NGC 5728 & $\left(\mathrm{R}_{1}\right) \mathrm{SB}\left(\underline{\mathrm{r}}^{\prime} 1, \mathrm{bl}, \mathrm{nr}, \mathrm{nb}\right) 0 / \mathrm{a}$ & 253 & 8.47 & 179 & 6.01 & 43.0 & 10.85 \\
\hline NGC 5750 & $(\mathrm{RL}) \mathrm{SAB}\left(\mathrm{r}^{\prime} 1 \underline{\mathrm{r}}, \mathrm{s}, \mathrm{bl}\right) 0 / \mathrm{a}$ & 149 & 3.79 & 153 & 3.90 & 60.2 & 10.74 \\
\hline NGC 5770 & $\mathrm{SAB}(\underline{\mathrm{r}} \mathrm{l}, \mathrm{bl}) 0^{+}$ & 345 & 4.03 & 132 & 1.55 & 22.4 & 10.10 \\
\hline NGC 5850 & $\left(\mathrm{R}^{\prime}\right) \mathrm{SB}(\mathrm{r}, \mathrm{bl}, \mathrm{nr}, \mathrm{nb}) \underline{\mathrm{a} b}$ & 260 & 10.46 & 139 & 5.59 & 36.0 & 10.77 \\
\hline NGC 5957 & $\left(\mathrm{R}^{\prime}\right) \mathrm{SA} \underline{\mathrm{B}}(\mathrm{rs}, \mathrm{bl}) \underline{\mathrm{a}} \mathrm{b}$ & 150 & 5.88 & 111 & 4.37 & 25.4 & 10.26 \\
\hline NGC 6014 & $\mathrm{SAB}(\mathrm{rs}, \mathrm{bl}) \underline{0} / \mathrm{a}$ & 191 & 3.92 & 119 & 2.45 & 35.3 & 10.33 \\
\hline NGC 7079 & (L)SAB $\underline{\mathrm{B}}_{\mathrm{a}}(\mathrm{s}, \mathrm{bl}) 0^{\circ}$ : & 253 & 3.64 & 165 & 2.38 & 51.3 & 10.64 \\
\hline \multicolumn{8}{|c|}{ Galaxies with $X$} \\
\hline ESO404-027 & $\mathrm{SAB}(\mathrm{s}) \underline{\mathrm{a}} \mathrm{b}:$ & 71 & 3.14 & 78 & 3.43 & 69.0 & 10.13 \\
\hline IC 1067 & $\mathrm{SB}(\mathrm{r}, \mathrm{bl}) \mathrm{b}$ & 175 & 3.34 & 91 & 1.74 & 38.3 & 9.91 \\
\hline IC 3806 & $\mathrm{SA}(\mathrm{r}) 0^{+}$ & 62 & 1.71 & 65 & 1.78 & 65.2 & 9.49 \\
\hline IC 4237 & $\mathrm{SB}(\mathrm{r}) \mathrm{b}$ & 87 & 2.21 & 135 & 3.41 & 46.2 & 10.53 \\
\hline IC 5240 & $\mathrm{SB}_{\mathrm{x}}(\mathrm{r}) 0 / \mathrm{a}$ & 170 & 4.80 & 122 & 3.44 & 45.0 & 10.45 \\
\hline NGC 0532 & $\mathrm{SAB}_{\mathrm{xa}}(\mathrm{r}) 0 / \mathrm{a}$ & 109 & 3.70 & 118 & 4.01 & 73.6 & 10.55 \\
\hline
\end{tabular}


Table 1

(Continued)

\begin{tabular}{|c|c|c|c|c|c|c|c|}
\hline Galaxy & Hubble Type & $\begin{array}{c}d V_{\text {cir }} / d r \\
\left(\mathrm{~km} \mathrm{~s}^{-1} \mathrm{kpc}^{-1}\right)\end{array}$ & $\begin{array}{c}d V_{\text {cir }} / d r \\
\text { normalized }\end{array}$ & $\begin{array}{c}V_{\max } \\
\left(\mathrm{km} \mathrm{s}^{-1}\right)\end{array}$ & $\begin{array}{c}h_{r} \\
(\mathrm{kpc})\end{array}$ & $\begin{array}{c}\text { INC } \\
\text { (degrees) }\end{array}$ & $\log _{10}\left(M^{*} / M_{\odot}\right)$ \\
\hline NGC 0615 & $\left(\mathrm{R}^{\prime}\right) \mathrm{SA}_{\mathrm{x}}(\mathrm{r}) \underline{\mathrm{a}} \mathrm{b}$ & 236 & 4.82 & 149 & 3.05 & 66.0 & 10.54 \\
\hline NGC 0779 & $(\mathrm{~L}) \mathrm{SA}_{\mathrm{x}}(\mathrm{rs}) \mathrm{a}$ & 175 & 2.50 & 130 & 1.86 & 70.3 & 10.35 \\
\hline NGC 1461 & $\mathrm{~S} \underline{\mathrm{A} B}(\mathrm{r}) 0^{\circ}$ & 231 & 3.90 & 131 & 2.21 & 70.8 & 10.40 \\
\hline NGC 1476 & $\operatorname{Im} \mathrm{sp}$ & 40 & 1.36 & 44 & 1.48 & 66.3 & 9.14 \\
\hline NGC 2654 & $\mathrm{SB}_{\mathrm{x}}(\mathrm{r}, \mathrm{nd}) 0 / \mathrm{a} \mathrm{sp}$ & 175 & 2.81 & 146 & 2.35 & 74.3 & 10.56 \\
\hline NGC 2683 & $\left(\mathrm{R}^{\prime} \mathrm{L}\right) \mathrm{SB}_{x a}(\mathrm{rs}) 0 / \mathrm{a} \mathrm{sp}$ & 236 & 3.30 & 163 & 2.29 & 74.9 & 10.62 \\
\hline NGC 3185 & $(\mathrm{RL}) \mathrm{SAB}_{\mathrm{ax}}(\mathrm{rs}, \mathrm{bl}) \mathrm{a}$ & 237 & 4.59 & 115 & 2.23 & 49.5 & 10.22 \\
\hline NGC 3254 & $\mathrm{SAB} \underline{B}_{x a} \mathrm{~b}$ & 111 & 5.64 & 106 & 5.40 & 73.3 & 10.57 \\
\hline NGC 3301 & $\left(\mathrm{R}^{\prime} \mathrm{L}\right) \mathrm{SAB}_{\mathrm{x}}(\mathrm{r}) 0^{+} \mathrm{sp}$ & 286 & 6.13 & 139 & 2.99 & 71.7 & 10.45 \\
\hline NGC 3380 & $(\mathrm{RL}) \operatorname{SAB}(\underline{\mathrm{r}} \mathrm{s}, \mathrm{bl}) 0 / \mathrm{a}$ & 204 & 3.98 & 102 & 1.99 & 20.8 & 9.96 \\
\hline NGC 3424 & $\mathrm{~S}_{\mathrm{x}} \underline{\mathrm{a}} \mathrm{b}: \mathrm{sp}$ pec & 173 & 3.42 & 143 & 2.83 & 75.8 & 10.43 \\
\hline NGC 3623 & $\left(\mathrm{R}^{\prime}\right) \mathrm{SAB}_{\mathrm{x}}(\mathrm{rs}) \mathrm{a}$ & 231 & 5.25 & 175 & 3.98 & 73.7 & 10.91 \\
\hline NGC 3673 & $\left(\mathrm{R}^{\prime}\right) \mathrm{SAB} \underline{B}_{x}(\mathrm{rs}) \mathrm{ab}$ & 166 & 4.65 & 122 & 3.41 & 52.6 & 10.51 \\
\hline NGC 3692 & $\left(\mathrm{R}^{\prime} \mathrm{L}\right) \mathrm{SA}(\mathrm{r}) 0 / \mathrm{a} \mathrm{sp}$ & 94 & 1.76 & 135 & 2.51 & 75.6 & 10.54 \\
\hline NGC 3887 & $(\mathrm{RL}) \mathrm{SA}_{\underline{B}_{x}}(\mathrm{rs}) \mathrm{bc}$ & 193 & 3.38 & 145 & 2.54 & 32.9 & 10.48 \\
\hline NGC 4123 & $\mathrm{SB}_{\mathrm{x}}(\mathrm{rs}) \mathrm{a} \underline{\mathrm{b}}$ & 259 & $10.42^{*}$ & 99 & 3.99 & 46.9 & 10.29 \\
\hline NGC 4192 & $\left(\mathrm{R}_{1}^{\prime}\right) \mathrm{SAB}_{\mathrm{x}}(\mathrm{rs}, \mathrm{nd}) \underline{\mathrm{a} b}$ & 270 & 13.06 & 140 & 6.79 & 72.0 & 10.77 \\
\hline NGC 4216 & $\left(\mathrm{R}_{2}^{\prime}\right) \mathrm{SAB}_{\mathrm{ax}}(\mathrm{r}, \mathrm{nd}) \mathrm{a} \mathrm{b}$ sp/E7-8 & 194 & 6.76 & 158 & 5.50 & 79.6 & 11.00 \\
\hline NGC 4220 & $(\mathrm{~L}) \mathrm{SAB}(\mathrm{r}) 0^{+}$ & 189 & 2.61 & 130 & 1.79 & 72.4 & 10.36 \\
\hline NGC 4235 & $\mathrm{~S}_{\mathrm{x}} 0^{+} \mathrm{sp}$ & 257 & 5.28 & 135 & 2.77 & 72.0 & 10.50 \\
\hline NGC 4268 & $\mathrm{SA} B(\underline{\mathrm{r}} \mathrm{s}) 0^{+}: \mathrm{sp}$ & 194 & 3.80 & 121 & 2.38 & 60.9 & 10.24 \\
\hline NGC 4293 & $\underline{\mathrm{R}}(\mathrm{L}) \mathrm{SB}_{\mathrm{x}}(\mathrm{r} \underline{\mathrm{s}}) 0 / \mathrm{a}$ & 313 & 9.15 & 119 & 3.48 & 62.1 & 10.42 \\
\hline NGC 4419 & $\mathrm{SA} \underline{B}_{x} 0 / \underline{\mathrm{a}} \mathrm{sp} / \mathrm{E} 6$ & 331 & 2.96 & 165 & 1.48 & 71.3 & 10.46 \\
\hline NGC 4462 & $\mathrm{SAB}_{\mathrm{x}}(\mathrm{rs}) \mathrm{a}$ & 188 & 3.70 & 137 & 2.69 & 63.3 & 10.54 \\
\hline NGC 4569 & $\left(\mathrm{R}^{\prime} \mathrm{L}\right) \mathrm{SA} \underline{B}_{\mathrm{x}}\left(\mathrm{rs}, \mathrm{x}_{1} \mathrm{r}\right) \mathrm{a}$ & 391 & 11.32 & 136 & 3.96 & 66.0 & 10.64 \\
\hline NGC 4586 & $\mathrm{SAB}_{x}(\mathrm{~s}, \mathrm{nd}) 0 / \mathrm{a} \mathrm{sp}$ & 218 & 6.32 & 90 & 2.62 & 69.8 & 10.13 \\
\hline NGC 4725 & $\left(\mathrm{R}^{\prime}\right) \mathrm{SAB}_{\mathrm{x}}(\mathrm{r}, \mathrm{nb}) \mathrm{a}$ & 366 & $10.90^{*}$ & 162 & 4.84 & 46.8 & 10.88 \\
\hline NGC 4818 & $(\mathrm{RL}) \mathrm{SA} \underline{B}_{\mathrm{xa}}(\mathrm{s}) 0 \circ$ & 342 & 7.52 & 141 & 3.10 & 67.2 & 10.48 \\
\hline NGC 4845 & $\left(\mathrm{R}^{\prime} \mathrm{L}\right) \mathrm{SAB}_{\mathrm{x}}\left(\mathrm{r}^{\prime} 1, \mathrm{nd}\right) 0 / \mathrm{a}$ & 262 & 10.59 & 135 & 5.44 & 75.1 & 10.55 \\
\hline NGC 4856 & (RL)SB0 ${ }^{-}$ & 277 & 5.75 & 172 & 3.58 & 68.7 & 10.74 \\
\hline NGC 4902 & $\mathrm{SB}(\underline{\mathrm{r}} \mathrm{s}, \mathrm{bl}) \mathrm{a} \underline{\mathrm{b}}$ & 206 & 4.23 & 209 & 4.30 & 21.5 & 11.05 \\
\hline NGC 5005 & $\left(\mathrm{R}_{2}{ }^{\prime}\right) \mathrm{SAB}_{\mathrm{xa}}(\mathrm{rs}) \mathrm{ab}$ & 396 & 5.99 & 244 & 3.70 & 66.7 & 11.10 \\
\hline NGC 5297 & $\mathrm{SAB}_{\mathrm{x}}(\mathrm{s}) \mathrm{bc} \mathrm{sp}$ & 78 & 2.57 & 126 & 4.16 & 73.6 & 10.60 \\
\hline NGC 5443 & $\left(\mathrm{R}^{\prime} \mathrm{L}\right) \mathrm{SAB}_{\mathrm{x}}(\mathrm{rs}) \mathrm{a} \mathrm{sp}$ & 139 & 5.08 & 119 & 4.36 & 68.0 & 10.49 \\
\hline NGC 5448 & $\left(\mathrm{R}_{1} \mathrm{~L}\right) \mathrm{SAB}_{\mathrm{x}}(\underline{\mathrm{r}} \mathrm{s}) \mathrm{a}$ & 167 & 8.43 & 143 & 7.24 & 65.4 & 10.77 \\
\hline NGC 5689 & $\left(\mathrm{R}^{\prime} \mathrm{L}\right) \mathrm{SAB}_{\mathrm{x}}\left(\mathrm{r}^{\prime} 1, \mathrm{nd}\right) \underline{0} / \mathrm{a}$ & 181 & 5.85 & 171 & 5.53 & 74.4 & 10.84 \\
\hline NGC 5757 & $\left(\mathrm{R}^{\prime}\right) \mathrm{SB}(\mathrm{rs}) \mathrm{ab}$ & 248 & 4.65 & 177 & 3.32 & 32.7 & 10.74 \\
\hline NGC 5806 & $\left(\mathrm{R}^{\prime} \mathrm{L}\right) \mathrm{SAB}(\mathrm{rs}, \mathrm{nrl}) \mathrm{ab}$ & 228 & 4.20 & 147 & 2.72 & 56.5 & 10.59 \\
\hline NGC 5854 & $(\mathrm{RL}) \mathrm{SAB}_{\mathrm{x}}(\mathrm{rl}) 0^{+} \mathrm{sp}$ & 189 & 3.76 & 119 & 2.37 & 71.1 & 10.23 \\
\hline NGC 5864 & $(\mathrm{RL}) \mathrm{SB}_{\mathrm{xa}} 0^{+} \mathrm{sp}$ & 139 & 2.48 & 120 & 2.14 & 71.6 & 10.35 \\
\hline NGC 5878 & $\mathrm{SAB}_{\mathrm{xa}}(\mathrm{rs}) \mathrm{ab}$ & 185 & 5.13 & 149 & 4.14 & 68.1 & 10.77 \\
\hline NGC 7140 & $\left(\mathrm{R}^{\prime}\right) \mathrm{SA} \underline{B}_{x}(\mathrm{rs}, \mathrm{nrl}) \mathrm{a} \underline{\mathrm{b}}$ & 161 & $7.39^{*}$ & 126 & 5.80 & 49.8 & 10.70 \\
\hline NGC 7163 & $\operatorname{SAB}_{x}(\mathrm{~s}) \mathrm{a}$ & 206 & 5.46 & 101 & 2.67 & 57.5 & 10.13 \\
\hline NGC 7171 & $\mathrm{SAB}_{x}(\mathrm{~s}) \mathrm{b}$ & 87 & 2.82 & 127 & 4.13 & 57.5 & 10.58 \\
\hline NGC 7179 & $\mathrm{SB}_{\mathrm{xa}}\left(\underline{\mathrm{r}}^{\prime} 1\right) 0 / \mathrm{a}$ & 100 & 2.48 & 135 & 3.33 & 58.9 & 10.60 \\
\hline NGC 7421 & $\left(\mathrm{R}^{\prime}\right) \mathrm{SB}(\mathrm{rs}, \mathrm{bl}) \mathrm{ab}$ & 132 & 2.93 & 102 & 2.26 & 27.9 & 10.15 \\
\hline NGC 7513 & $\left(\mathrm{R}^{\prime} \mathrm{L}\right) \mathrm{SB}(\mathrm{rs}) \mathrm{a}$ & 102 & 3.09 & 101 & 3.05 & 48.4 & 10.21 \\
\hline NGC 7531 & $\mathrm{SAB}_{\mathrm{x}}(\mathrm{r}) \mathrm{a}$ & 221 & 3.90 & 144 & 2.55 & 57.5 & 10.44 \\
\hline PGC045650 & $\mathrm{SAB} \underline{a}_{a}(\mathrm{~s}) \mathrm{ab}$ & 185 & 2.38 & 136 & 1.76 & 72.7 & 10.37 \\
\hline
\end{tabular}

Note. The inner slope, $d V_{\text {cir }} / d r$, and the maximum velocity, $V_{\max }$, are calculated from the $\mathrm{S}^{4} \mathrm{G} 3.6 \mu \mathrm{m}$ images. The stellar masses $M_{*}$ are from Munoz-Mateos et al. (2015), and the disk scale length and inclination are from Salo et al. (2015). Normalized $d V_{\text {cir }} / d r$ indicates scaling of the inner slope with $V_{\max } / h_{r}$ : the $\mathrm{X}$-shaped galaxies with $i<60^{\circ}$ and normalized $d V_{\text {cir }} / d r>8$ are marked with an asterisk. The barlens classifications are from Buta et al. (2015) and Laurikainen et al. (2011), and the X classifications are from Laurikainen \& Salo (2016b).

major axis, traces the observed distribution of apparent sizes (normalized to the bar size) and aspect ratios of barlens and X features versus the parent galaxy inclination. The same model also reproduces the observed dependence of barlens boxiness parameter on galaxy inclination: for $i \lesssim 40^{\circ}$, barlenses have
$B_{4}>0$, but for larger inclination, $B_{4}$ gets negative, that is, isophotes become boxy. This is in line with Erwin \& Debattista (2013), who suggested that $\mathrm{B} / \mathrm{P}$ bulges can be identified at intermediate inclinations $\left(i=40-60^{\circ}\right)$ by the boxy inner isophotes of bars. 

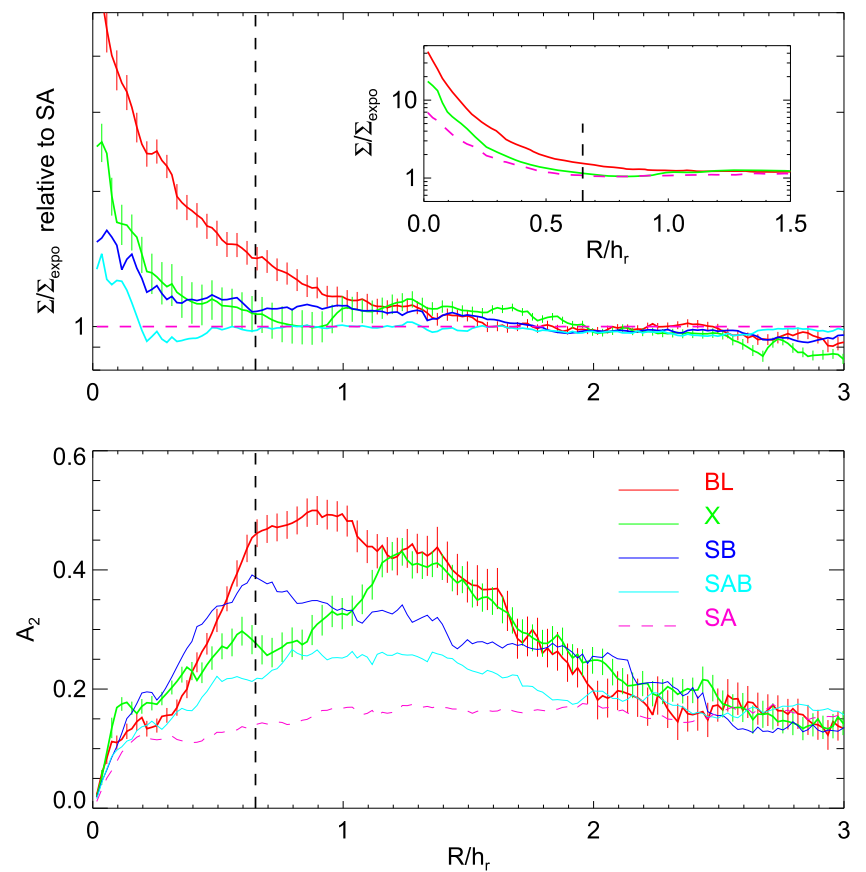

Figure 7. The insert in the upper frame shows the median-stacked surface brightness excess of barlens, X-shaped, and SA galaxies, relative to their exponential outer disk profiles extrapolated to the center. Decomposition parameters for the disks are from Salo et al. (2015), and the bar family classification is from Buta et al. (2015). In the large frame, the excess brightness of barlens (bl) and X samples is shown relative to SA galaxies. For comparison, samples of SB and SAB are also included (all samples are limited to galaxies with $M^{*}>10^{10} M_{\odot}, T \leqslant 5$ ). The lower frame indicates the medians of $A_{2}$ Fourier-amplitude profiles. The vertical dashed line indicates the median barlens size relative to disk scale length $\left(r / h_{r} \approx 0.65\right.$; from HerreraEndoqui et al. 2015).

\section{Discussion}

The preceding simulations and comparisons to observations suggest that a steep inner rotation curve slope favors a pure barlens face-on morphology, characterized by a nearly round central appearance. With a shallower rotation curve the orbits in the central regions become more elongated along the bar major axis, leading to an oval-shaped bar, or even to an Xshaped face-on feature (narrow waist in the minor axis).

The strong constraint on the simulated inner slope likely explains why barlenses, which are fairly conspicuous observationally, have not been encountered in simulation studies before Laurikainen et al. (2014) and Athanassoula et al. (2015): typically the classical bulge components employed, if included at all, have been less centrally concentrated than those in the current simulations. In Debattista et al. (2004), the problem of not attaining round bar-related bulges in simulations was acknowledged, and it was speculated that extended central objects with $B / D=0.1-0.2$ might be needed, based on the simulations of Shen \& Sellwood (2004) that studied the robustness of bars against central black hole masses. In Shen \& Sellwood (2004) simulations, a rigid halo was assumed; however, very similar conclusions were reached in Athanassoula et al. (2005) using live halos. Our current simulations demonstrate that a substantially smaller $B / D$ (of a few percents) is sufficient, provided that realistic, small, effective bulge radii from observations $\left(r_{\text {eff }} / h_{r} \lesssim 10 \%\right)$ are adopted. Such central mass concentrations can affect the orbital morphology up to $\sim 10 r_{\text {eff }}$, while still not endangering the survival of the narrow bar. Simulating steep central slopes implies a need for small gravity softening: in particular, cosmological simulations so far lack the resolution necessary to resolve the inner bar dynamics (see, e.g., the discussion in Brooks \& Christensen 2016).

We have demonstrated that the inclusion of a small classical bulge in simulations can reproduce the barlens morphology. Does this imply that the observed barlens galaxies harbor classical bulges that were in place before the bar formed? An alternative is that the central mass concentrations inside the barlens region have formed together with the bar, via barinduced inflow and star formation. Namely, barlenses are typically associated with strong bars, and with galaxies that have consumed most of their gas. Such inflow was also the mechanism that created the central mass concentrations in the Athanassoula et al. (2015) simulations, which did not include any initial classical bulge component. Although most of the simulated galaxies had traces of $\mathrm{X}$ shapes in their face-on morphology, it is feasible that a somewhat stronger inflow in their models could have created even steeper central rotation slopes and thus more realistic pure barlens morphologies.

Observational evidence for the possible bar-related growth is provided by Figure 7, which compares the amount of central density excess in barlens galaxies to other types of barred galaxies (with $\mathrm{X}$, and with those $\mathrm{SB}, \mathrm{SAB}$ galaxies that do not have barlens or X morphology), and to nonbarred SA galaxies. Clearly, barlens galaxies have the strongest central excess (consistent with their early $T$ types), both at the region corresponding to the barlens itself, $r / h_{r} \sim 0.5-0.8$, and in the very center, $r / h_{r}<0.2$. Among the different galaxy families, the central excess correlates with the strength of the bar (or non-axisymmetric perturbation in general, here measured in terms of $A_{2}$ Fourier amplitudes of surface density), supporting the role of bars in building the central concentration. A similar conclusion was reached recently by Díaz-García et al. (2016a) when they compared SB, SAB, and SA galaxies; however, the difference is far more pronounced when barlens galaxies are considered.

Nevertheless, the above link between strong bars and central concentrations still leaves open the possibility that the observed central components represent classical bulges, which have helped the bars to gain strength by removing the angular momentum of the bars. Such dependence was seen, for example, in the simulations of Athanassoula \& Misiriotis (2002), where the bar $A_{2}$ maximum was nearly doubled in a model that included a classical bulge component, compared to a model with only disk and halo components. However, the classical bulge in those simulations was quite extended and massive, with $B / D=0.60$. In the current simulations with small compact classical bulges, the strength of the bar is practically independent of the adopted initial $B / D$, so no similar straight-forward conneection can be made. Clearly, spectroscopic observations of the stellar ages and metallicities are needed to distinguish between the disky pseudobulge/classical bulge character of the central concentrations in barlens galaxies.

\section{Conclusions}

The barlens-sized central light concentrations in early-type disk galaxies are often identified as massive classical bulges. In this paper we have provided further evidence that, at least in the case of barred galaxies, they represent $\mathrm{B} / \mathrm{P} / \mathrm{X} / \mathrm{bl}$ structures related to the secular evolution of bars. However, the exact 
morphology at low galaxy inclination depends on the galaxy mass distribution.

Our main conclusions are the following.

(1) Stellar dynamical simulations, using parameter values appropriate for MW mass galaxies, indicate that a steeply rising inner rotation curve is required for obtaining a pure barlens morphology (no trace of $\mathrm{X}$ in the low inclination view). In the current simulations, this was achieved by including a small classical bulge with the initial values, or by using a sufficiently centrally concentrated halo. The threshold inner slope in simulations is $d V_{\text {cir }} / d r \sim$ $5 V_{\max } / h_{r}$.

(2) The simulated behavior is in qualitative agreement with slopes derived from $\mathrm{S}^{4} \mathrm{G} 3.6 \mu \mathrm{m}$ images, which indicate that barlens galaxies have steeper baryonic rotation curves $\left(d V_{\text {cir }} / d r \gtrsim 250 \mathrm{~km} \mathrm{~s}^{-1} \mathrm{kpc}^{-1}\right)$ than the galaxies that exhibit $\mathrm{X}$ signature, even at $i<60^{\circ}$.

(3) Comparisons of stacked surface density profiles indicate that barlens galaxies have larger $A_{2}$ amplitudes than any other type of barred galaxies. They also have the largest relative excess of inner surface density (within $r / h_{r}<0.2$ ), which can be interpreted as evidence for bar-induced inflow in the past.

We thank the referee for useful suggestions on how to clarify the presentation of the results. This work was supported by the DAGAL network: People Programme (Marie Curie Actions) of the European Unions Seventh Framework Programme FP7/ 2007-2013 under REA grant agreement number PITN-GA2011-289313. The grant from Academy of Finland (No. 297738) is also acknowledged.

\section{References}

Athannasoula, E. 2016, in Galactic Bulges, Vol. 418, ed. E. Laurikainen, R. Peletier, \& D. Gadotti (Berlin: Springer), 391

Athanassoula, E., Lambert, J. C., \& Dehnen, W. 2005, MNRAS, 363, 496

Athanassoula, E., Laurikainen, E., Salo, H., \& Bosma, A. 2015, MNRAS, 454,3845

Athanassoula, E., Machado, R. E. G., \& Rodionov, S. A. 2013, MNRAS, 429, 1949
Athanassoula, E., \& Misiriotis, A. 2002, MNRAS, 330, 35

Athanassoula, E., Rodionov, S. A., Peschken, N., \& Lambert, J. C. 2016, ApJ, 821,90

Brooks, A., \& Christensen, C. 2016, in Galactic Bulges, Vol. 418, ed E. Laurikainen, R. Peletier, \& D. Gadotti (Berlin: Springer), 317

Bureau, M., Aronica, G., Athanassoula, E., et al. 2006, MNRAS, 370, 753

Buta, R., Kartik, S., Athanassoula, E., et al. 2015, ApJS, 217, 32

Combes, F., Debbasch, F., Friedli, D., \& Pfenniger, D. 1990, A\&A, 233, 81

Courtois, H. M., Tully, R. B., Fisher, J. R., et al. 2009, AJ, 138, 1938

Debattista, V. P., Carollo, C. M., Mayer, L., \& Moore, B. 2004, ApJL, 604, L96

Debattista, V. P., Mayer, L., Carollo, C. M., et al. 2006, ApJ, 645, 209

Díaz-García, S., Salo, H., \& Laurikainen, E. 2016a, A\&A, 596, A84

Díaz-García, S., Salo, H., Laurikainen, E., \& Herrera-Endoqui, M. 2016b, A\&A, 7, A160

Di Matteo, P., Haywood, M., Combes, F., Semelin, B., \& Snaith, O. N. 2013, A\&A, 553, A102

Erroz-Ferrer, S., Knapen, J. H., Leaman, R., et al. 2016, MNRAS, 458, 1199

Erwin, P., \& Debattista, V. 2013, MNRAS, 431, 3060

Herrera-Endoqui, M., Díaz-García, S., Laurikainen, E., \& Salo, H. 2015, A\&A, 582, A86

Herrera-Endoqui, M., Salo, H., Laurikainen, E., \& Knapen, J. 2016, A\&A, in press (arXiv:1610.02330)

Kuijken, K., \& Dubinski, J. 1995, MNRAS, 277, 1341

Laurikainen, E., \& Salo, H. 2002, MNRAS, 337, 1118

Laurikainen, E., \& Salo, H. 2016a, in Galactic Bulges, Vol. 418, ed E. Laurikainen, R. Peletier, \& D. Gadotti (Berlin: Springer), 77

Laurikainen, E., \& Salo, H. 2016b, A\&A, in press (arXiv:1609.01936)

Laurikainen, E., Salo, H., Athanassoula, E., et al. 2014, MNRAS, 444, L80

Laurikainen, E., Salo, H., Buta, R., \& Knapen, J. H. 2007, MNRAS, 381, 401

Laurikainen, E., Salo, H., Buta, R., \& Knapen, J. H. 2011, MNRAS, 418, 1452

Lelli, F., Fraternali, F., \& Verheijen, M. 2013, MNRAS, 433, L30

Lütticke, R., Dettmar, R.-J., \& Pohlen, M. 2000, A\&AS, 145, 405

Minchev, I., Famaey, B., Quillen, A., et al. 2012, A\&A, 548, A126

Munoz-Mateos, J.-C., Carlos, J., Sheth, K., et al. 2015, ApJS, 219, 3

Patsis, P. A., Skokos, Ch., \& Athanassoula, E. 2002, MNRAS, 337, 578

Pfenniger, D., \& Friedli, D. 1991, A\&A, 252, 75

Querejeta, M., Meidt, S. E., Schinnerer, E., et al. 2015, ApJS, 219, 4

Quillen, A. C., Minchev, I., Sharma, S., et al. 2014, MNRAS, 437, 1284

Raha, N., Sellwood, J. A., James, R. A., \& Kahn, F. D. 1991, Natur, 352, 411

Saha, K., Martinez-Valpuesta, I., \& Gerhard, O. 2012, MNRAS, 421, 333

Salo, H., Laurikainen, E., Laine, J., Comeron, S., et al. 2015, ApJS, 219, 4

Salo, H., Rautiainen, P., Buta, R., et al. 1999, AJ, 117, 792

Shen, J., \& Sellwood, J. A. 2004, ApJ, 604, 614

Sheth, K., Regan, M., Hinz, J. L., et al. 2010, PASP, 122, 1397

Springel, V., \& Hernquist, L. 2005, ApJL, 622, L9 